\title{
Relato de experiência da Implantação da capacitação sobre imunização do estado do Amazonas por meio do Telessaúde
}

\author{
Experience report of the implementation of training on immunization of the state of \\ Amazonas through Telehealth
}
Informe de experiencia de la implementación de la capacitación en inmunización del estado de Amazonas a través de Telesalud

Josy Lira Dias ${ }^{1 *}$, Mitsi Silva Moisés ${ }^{1}$, Maria Izabel Nogueira do Nascimento ${ }^{1}$, Katheuce Nunes da Silva ${ }^{1}$, Maria Graciede Filha Santarém Andrade'1.

\section{RESUMO}

Objetivo: Descrever a experiência que foi vivida durante a implantação da capacitação sobre imunização por meio do Telessaúde no estado do Amazonas em 2020. Relato de experiência: Trata-se de um estudo do descritivo, do tipo relato de experiência, realizado a partir da vivência da implantação da capacitação sobre imunização fazendo uso da ferramenta Telessaúde no estado do Amazonas. Essa implantação faz parte de um projeto de doutorado que teve a sua liberação aprovada pelo comitê de ética em pesquisa. Foram realizadas 7 palestras no período de setembro a novembro de 2020, com temas sobre imunologia, conceito de vacinas e como elas agem no organismo. Sendo abordado os calendários vacinais ofertados pelo Programa Nacional de Imunizações, enfatizando as modificações e introduções de novas vacinas. Destacando sobre a sala de vacina e suas especificidades e normativas, vacinas especiais e fluxo de pedido de vacinas. Considerações finais: Esta capacitação apresentou um grande alcance no estado do Amazonas, onde as equipes de sala de vacina tiveram a oportunidade de atualizar seus conhecimentos e tirar as suas dúvidas. Tendo como intuito a melhoria das ações em saúde voltadas para a área de vacinação, quando as equipes são capacitadas em assuntos específicos.

Palavras-chave: Imunização, Telemedicina, Capacitação de recursos humanos em saúde.

\begin{abstract}
Objective: Describe the experience that was lived during the implementation of training on immunization through Telehealth in the state of Amazonas in 2020. Experience report: This is a descriptive study, of the experience report type, carried out from the experience of implementing training on immunization using the Telehealth tool in the state of Amazonas. This implementation is part of a doctoral project that had its release approved by the research ethics committee. Seven lectures were held from September to November 2020, with themes on immunology, the concept of vaccines and how they act in the body. Being addressed the vaccine calendars offered by the National Immunization Program, emphasizing the modifications and introductions of new vaccines. Highlighting about the vaccine room and its specificities and regulations, special vaccines and vaccine order flow. Final considerations: This training had a wide reach in the state of Amazonas, where the vaccination room teams had the opportunity to update their knowledge and answer their questions. With the aim of improving health actions aimed at the vaccination area, when teams are trained in specific subjects.
\end{abstract}

Key words: Immunization, Telemedicine, Health human resource training.

${ }^{1}$ Fundação de Vigilância em Saúde do Amazonas (FVS-AM), Manaus - AM. *E-mail: joliradias@hotmail.com SUBMETIDO EM: 2/2021

PUBLICADO EM: $3 / 2021$ 


\section{RESUMEN}

Objetivo: Describir la experiencia que se vivió durante la implementación de la capacitación en inmunización a través de Telesalud en el estado de Amazonas en 2020. Informe de experiencia: Se trata de un estudio descriptivo, del tipo informe de experiencia, realizado a partir de la experiencia de implementar capacitaciones en inmunización utilizando la herramienta Telesalud en el estado de Amazonas. Esta implementación es parte de un proyecto de doctorado cuyo lanzamiento fue aprobado por el comité de ética en investigación. Se realizaron siete conferencias de septiembre a noviembre de 2020, con temas sobre inmunología, el concepto de vacunas y cómo actúan en el organismo. Se abordaron los calendarios de vacunas que ofrece el Programa Nacional de Inmunizaciones, destacando las modificaciones e introducciones de nuevas vacunas. Destacando sobre la sala de vacunas y sus especificidades y regulaciones, vacunas especiales y flujo de pedidos de vacunas. Destacando en la sala de vacunas y sus especificidades y regulaciones, vacunas especiales y flujo de pedidos de vacunas. Consideraciones finales: Esta capacitación tuvo un amplio alcance en el estado de Amazonas, donde los equipos de la sala de vacunación tuvieron la oportunidad de actualizar sus conocimientos y responder a sus preguntas. Con el objetivo de mejorar las acciones de salud dirigidas al área de vacunación, cuando los equipos están capacitados en temas específicos.

Palabras clave: Inmunización, Telemedicina, Capacitación de recursos humanos en salud.

\section{INTRODUÇÃO}

O Telessaúde faz uso das Tecnologias de Informação e Comunicação (TIC's), buscando diminuir as distâncias e integrar os serviços de saúde, trabalhando o aperfeiçoamento das equipes de saúde (OPAS, 2016). No Brasil o cenário dos serviços de saúde apresenta uma complexidade gerando novas estratégias e ferramentas como o Telesssaúde, para oportunizar de maneira mais equitativa em todo o país uma capacitação com informações atualizadas para que a população seja atendida de modo eficaz e eficiente (REZENDE EJC, et al., 2010).

Como os profissionais de saúde são essenciais para o Sistema Único de Saúde (SUS), não somente como serviço de atendimento ao público mais também como gestão, onde a capacitação deve ter uma ampla visão para que haja uma modificação das ações em saúde coletiva (CECCIM RB, et al., 2009). O Telessaúde por meio da educação são serviços que tem importância para que os trabalhadores do SUS tenham condições de realizar capacitações em suas áreas, avaliando e refletindo suas ações tendo como base as necessidades da população (SILVA TPS, et al., 2020).

Os calendários de vacinação têm variação devido aos estudos realizados sobre a quantidade de doses das vacinas, a situação epidemiológica vigente no país, a viabilidade da introdução de novas vacinas e a avaliação da ampliação das faixas etárias. A normatização é atualizada frequentemente e essas modificações necessitam de constante capacitação para disseminar a informação aos profissionais (BRITO MFP, et al., 2014).

O Programa Nacional de Imunizações (PNI) teve seu início no ano de 1973 por maio do Ministério da Saúde e que vem abrangendo toda a população do recém-nascido ao idoso, indígenas e pessoas que necessitam de imunobiológicos especiais através dos Centros de Referência de Imunobiológicos Especiais (CRIEs), este programa tem tido êxito na diminuição de doenças imunopreveníveis no Brasil, atuando de maneira preventiva por meio da utilização de vacinas (ANDRADE RS, et al., 2014).

Tem sido constatado que os erros em imunização acontecem devido à escassez ou falta de capacitação dos profissionais que atuam em sala de vacina, gerando situações como dificuldade em repassar informações exatas sobre as vacinas, os erros vacinais, o crescimento do quantitativos de eventos adversos, isso tem dado acesso a introdução dos mitos e fake news. A população fica apreensiva em tomar as vacinas, gerando atrasos vacinais e recusas, diminuindo as coberturas vacinais e deixando pessoas susceptíveis possibilitando a reintrodução de doenças imunopreveníveis (MARTINS KM, et al., 2019).

No estado do Amazonas, de acordo com o Instituto Brasileiro de Geografia e Estatística (IBGE), destacam que é o maior estado em extensão entre as 27 unidades federativas do Brasil, apresentando uma área de 
1.559.148,89 $\mathrm{Km}^{2}$, no censo realizado em 2010 foi descrito que a população era de 3.483 .985 habitantes, o Amazonas apresenta 62 municípios, onde a capital Manaus onde se localiza a maior parte da população do estado com cerca de 52,25\% (SECOM, 2016).

Ao identificar a problemática no serviço de saúde, pode ser avaliado a conexão da teoria e a prática, realizando uma melhoria na comunicação dentro da equipe de saúde, com a troca de experiências de modo a trabalhar as opiniões, porém no âmbito da saúde ao utilizar o Telessaúde para educação contínua das normas atuais e ainda responder a questionamentos e dúvidas existentes (BROWN D, et al., 2018).

Este artigo tem como objetivo descrever a experiência que foi vivida durante a implantação da capacitação sobre imunização por meio do Telessaúde no estado do Amazonas em 2020.

\section{RELATO DE ESPERIÊNCIA}

Este relato de experiência foi realizado a partir da vivência da implantação da capacitação sobre imunização por meio do Telessaúde no estado do Amazonas. Esta capacitação sobre imunização teve ampla divulgação nas redes sociais por meio do Telessaúde e do Programa Estadual de Imunização.

Foi realizada por meio dos polos de Telessaúde de cada município, também pelo canal do Telessaúde no YouTube, através do aplicativo Mano e o mesmo podendo ser acessado através do celular ou do computador, também foi disponibilizado por meio do Programa IPTV (Internet Protocol Television) que é a transmissão de um sinal de TV via pela internet com conexões de banda larga. Dessa forma, buscando facilitar o acesso das atualizações em imunização para todas as equipes de saúde que trabalham em sala de vacina ou tem interesse em aprender sobre $o$ assunto.

Essa implantação faz parte de um projeto de doutorado que teve a sua liberação aprovada pelo comitê de ética em pesquisa $n^{\circ} 4.186 .915$ do projeto de pesquisa "O Telessaúde como provedor do conhecimento e qualificação sobre a imunização no estado do Amazonas no contexto da pandemia do Coronavírus". A experiência de implantação da capacitação fazendo uso das Webpalestras constituiu-se como uma iniciação a um novo modelo de trabalho, ao qual teve uma grande participação dos municípios por meio das equipes de sala de vacina.

As atividades ocorreram no município de Manaus-AM, no período de setembro a novembro de 2020 e teve início com a Palestra no dia 16.09.2020 sobre "Histórico do Programa de Imunização", para o início da capacitação e para abordar sobre o programa de imunização no Brasil e no estado do Amazonas, a coordenadora do programa estadual de imunização a enfermeira Maria Izabel Nogueira do Nascimento abordou sobre a evolução do calendário vacinal e as dificuldades encontradas desde o ano de 2015 até o momento em alcançar as coberturas vacinais adequadas, destacando o momento epidemiológico pandêmico do Coronavírus. Teve uma duração de 2:00h e grande participação.

No dia 30.09.2020 foi realizado a palestra sobre "Noções básicas de imunologia, de vacinas, cadeia e rede de frio", ao realizar a palestra de capacitação, foi observado que alguns municípios estavam tendo dificuldade com a conexão para assistir a aula, devido principalmente a questão climática apresentando muita chuva e causando interferências. Além do assunto abordado foi falado sobre a finalização da campanha da influenza que estava finalizando no dia da capacitação. A palestra teve uma duração de 1:45h, deixando 15 minutos para perguntas e respostas.

A terceira palestra ocorreu no dia 08.10 .2020 sobre "Calendário básico de vacinação da criança", devido à grande quantidade de vacinas da criança foi utilizado o tempo de 1:50h, tendo 10 minutos para perguntas e respostas, mesmo com o pouco tempo para as perguntas, foi bem utilizado. Houve alguns problemas relatados sobre a dificuldade de acesso devido a problemas com a internet, no Polo do Telessaúde também teve uma pequena pausa devido a problemas com a internet, mais logo retornou sem mais intercorrências.

Foi realizada a palestra sobre "SPNI e Campanhas" no dia 15.10.2020, nesta data em alguns municípios estava sendo considerado ponto facultativo devido a data do dia do professor, esses municípios optaram por assistir a Webpalestra via aplicativo Mano. A palestra teve duração de 1:00h e houve o tempo de perguntas e respostas de 30 minutos devido ao momento de campanha de Multivacinação e contra a Poliomielite e assim tirando as dúvidas sobre o sistema de informação e da forma de informação da campanha. 
A palestra sobre "Calendário de vacinação do adolescente, do adulto, gestante e do idoso" ocorreu no dia 22.10.2020, esta palestra teve a duração de 1:00h com tempo de 30 minutos para os questionamentos, aos quais tiveram muitas dúvidas, principalmente quanto ao calendário do adolescente.

O tema abordado na palestra do dia 11.11.2020 foi sobre o "CRIE, os imunobiológicos especiais, eventos adversos pós-vacinação (leves, moderados e graves)" foi apresentada em 1:30h, tendo 30 minutos para perguntas e respostas. Teve um momento de falta de energia no polo de Telessaúde que interrompeu por alguns instantes a transmissão. Ao ser descrito Centro de Referência de Imunobiológicos Especiais (CRIE), foi descrito o local onde funciona o serviço, ao demonstrar as vacinas que podem ser administradas da rotina para pacientes que tem indicação de acordo com cada necessidade, chamou atenção a documentação necessária para que possam administrar as doses e que todos esses casos devem ser repassados primeiramente para o CRIE para serem autorizados, assim como as vacinas especiais.

$\mathrm{Na}$ última palestra que teve a sua realização no dia 25.11.2020 sobre "Sala de vacina e a rotina de avaliação das estatísticas do Programa estadual de imunização do Amazonas", foi realizada em 1:30h, sendo trabalhadas as dúvidas nos 30 minutos restantes, depois foi realizado o agradecimento a todos pela a participação e finalizando assim a série de palestras propostas de capacitação em sala de vacina no estado do Amazonas no ano de 2020.

Ao final de cada palestra foi emitido um código para que os participantes pudessem anotar e enviar ao Telessaúde junto com o nome ou a frequência para que pudessem ter a sua certificação. Os certificados foram disponibilizados no site do Telessaúde do Amazonas e estariam disponíveis durante três meses para serem acessados pelos referidos participantes. Todas as palestras ficam disponíveis no canal do Telessaúde no YouTube e no site do Telessaúde do Amazonas para que sejam disponibilizadas para aqueles que não conseguiram assistir nos dias pré-agendados.

\section{DISCUSSÃO}

Ao montar o projeto para realizar a capacitação por meio do Telessaúde para ser realizado em um estado com grandes distancias, foi um grande desafio em divulgar e buscar que todas as equipes que trabalham em sala de vacina pudessem ter essa oportunidade para trabalhar novos conhecimentos específicos no estado do Amazonas.

Para Brasil (2012) tem ocorrido uma melhoria no processo de capacitação dos profissionais da área de saúde resultantes da implantação e trabalho realizado por meio do Programa Telessaúde no país, principalmente para os profissionais que moram e trabalham em localidades distantes ou de difícil acesso e que não poderiam realizar a atualização de outra maneira.

Ao empreender por meio do Telessaúde em realizar uma capacitação em sala de vacina no estado, foi observado que as pessoas conseguem acompanhar o uso das tecnologias propostas, a dificuldade encontrada foi o sinal da internet em alguns municípios e com fortes chuvas principalmente no mês de novembro de 2020 , foram pontos que influenciaram as equipes a assistirem as palestras em outro momento com melhor sinal.

Esta EPS é importante para que o profissional entenda a relação entre as ações realizadas no atendimento com a necessidade da população, levando a uma compreensão crítica das ações realizadas, do procedimento e da proatividade dentro do serviço de saúde. O Programa de Telessaúde no Brasil teve o seu início objetivando desenvolver a EPS com as equipes que trabalham na atenção básica em saúde, de maneira inovadora, onde as equipes de saúde por meio da utilização de novas ferramentas (BROWN D, et al., 2018).

A organização e elaboração das palestras foi um momento de aprendizado para toda a equipe, buscando destacar as principais necessidades do público-alvo e se adaptar a este novo momento de distanciamento social e da utilização das novas ferramentas objetivando alcançar o maior número de pessoas que trabalha em sala de vacina e atualizar seus conhecimentos na área específica. Teve nervosismo em não estar interagindo com os participantes presencialmente o que era o rotineiro trabalho de capacitação e a questão da câmera, foram pontos podemos destacar como superados pelo grupo. 
Ao fazer uso das novas tecnologias de informática voltado a saúde é um avanço para apoiar o profissional na atuação diária, com atualizações constantes de seus conhecimentos e dessa forma aprimorando as ações realizadas voltadas para o aumento das coberturas vacinais. Realizando uma contribuição por meio de informações sobre o serviço de saúde favorecendo a associação entre a parte administrativa e a clínica da unidade (OPAS, 2001).

Mesmo que a utilização de tecnologia para capacitar profissionais que trabalham com a saúde não seja algo novo, ainda verificamos muitas dificuldades em aceitação da ferramenta antes do período de pandemia do COVID-19 com o distanciamento social e a dificuldade em ter cursos presenciais. Em meio a tantas dificuldades, houve uma mudança de atitude e pensamentos, o que fez com que tivesse uma grande aceitação por parte de toda a equipe e dos participantes.

Existem poucas publicações científicas sobre o domínio e embasamento dos profissionais que trabalham em sala de vacina e são menores ainda as questões associadas com a vacinação e normas do PNI. A EPS voltada para a sala de vacina precisa ter uma permanência para uma qualificação de qualidade de toda a equipe, tendo em vista a avanço das ações fundamentado em conhecimento atualizado (MARINELLI NP, et al., 2015).

Para que ocorra uma assistência de melhor qualidade para a saúde coletiva a OMS (2009), destaca que uma das formas é buscar trabalhar um aperfeiçoamento no acesso as informações e atualizações com ferramentas para a área de saúde atuar com qualidade. Ao introduzir as novas tecnologias como estratégia e buscando realizar um planejamento estratégico para que todos os profissionais que atuam na área de saúde possam ter acesso à informação.

Mesmo com pontos a serem superados, finalizamos a implantação da capacitação por meio do Telessaúde e ficamos animadas e com vontade de manter o trabalho por meio dessa ferramenta, uma parceria que teve uma ótima aceitação por parte do grupo como pelos participantes. Pretendemos continuar trabalhando no próximo ano com esta e outras ferramentas digitais e assim contribuindo com conhecimentos específicos das equipes de sala de vacina como também a inclusão digital em todos os municípios do estado.

As TIC's têm sido trabalhadas para treinamento e de informação em saúde para provedores para trabalhadores da assistência multidisciplinares, assim como quem tiver interesse nas apresentações que ficam gravadas e disponibilizadas gratuitamente, ampliando assim o campo de atuação do Telessaúde (CAETANO R, et al., 2020). Tem-se trabalhado com o termo e-saúde (eHealth) que se refere a saúde digital, que dentro do campo da informação e da ação a tecnologia digital possa ser utilizada com à intenção de melhorar a saúde como um todo (OMS, 2019).

O Amazonas é o maior estado brasileiro e a tecnologia por meio dos serviços do Programa de Telessaúde vem contribuir para reduzir as desigualdades de oportunidade em realizar atualizações que possam abranger toda equipe de saúde. Além de solucionar as barreiras geográficas e trabalhar o distanciamento social com a utilização de tecnologia como ferramenta no avanço da formação de profissionais da área de saúde que trabalham na sala de vacina ou tem interesse em conhecer mais.

Esta capacitação teve uma grande adesão por parte dos profissionais de sala de vacina, abarcando todos os municípios do estado e contribuindo para a melhoria das ações em saúde. Dessa maneira a equipe pode participar da capacitação sem necessitar sair do seu local de trabalho e sem custos adicionais tirando suas dúvidas em tempo real. Uma das dificuldades encontradas foi a baixa qualidade da internet em alguns locais principalmente no interior do estado e o mal tempo dificultando a transmissão.

\section{AGRADECIMENTOS}

Agradecemos a equipe do Programa Estadual de Imunização do Estado do Amazonas pelo empenho em realizar a implantação da capacitação utilizando um novo modelo de trabalho que são as Webpalestras. A equipe do Polo de Telessaúde do estado do Amazonas foi essencial durante todo o processo de trabalho, nos apoiando na divulgação, assessoria para que a implantação fosse realizada. Agradecemos aos coordenadores de imunização dos municípios e suas equipes que estiveram conosco para trabalharmos a divulgação do conhecimento sobre imunização no estado. 


\section{REFERÊNCIAS}

1. ANDRADE RS, et al. Conhecimento De Mães Sobre O Calendário de vacinação e fatores que levam ao atraso vacinal infantil. Cogitare Enfermagem 2014; 19 (1): 94-100.

2. BRASIL. Ministério da Saúde. Manual de Telessaúde para Atenção Básica/Atenção Primária à Saúde/Ministério da Saúde, Universidade Federal do Rio Grande do Sul. - Brasília: Ministério da Saúde, 2012. 123 p: il. - (Série A. Normas e Manuais Técnicos). Disponível em: http://189.28.128.100/dab/docs/portaldab/publicacoes/manual_telessaude.pdf. Acessado em: 10 de agosto de 2020.

3. BRITO MFP, et al. Caracterização das notificações de procedimentos inadequados na administração de imunobiológicos em Ribeirão Preto, São Paulo, 2007-2012. Epidemiol Serv Saúde. 2014; 23 (1):33-44.

4. BROWN D, et al. Utilização do telessaúde na educação permanente pelos enfermeiros da Estratégia da Saúde da Família. Enfermagem Brasil, 2018;17(4):326-335.

5. CAETANO R, et al. Desafios e oportunidades para telessaúde em tempos da pandemia pela COVID-19: Uma reflexão sobre os espaços e iniciativas no contexto brasileiro. Cad Saúde Pública 2020;36(5):1-16.

6. CECCIM RB, et al. Educação na saúde, saúde coletiva e ciências políticas: uma análise da formação e desenvolvimento para o Sistema Único de Saúde como política pública. Lugar Comum UFRJ. 2009; 2 8(1): $159-80$.

7. MARINELLI NP, et al. Conhecimento dos profissionais de enfermagem em sala de vacina: análise da produção científica. Rev Univap. 2015; 21 (38): 2237-53.

8. MARTINS KM, et al. A importância da imunização: revisão integrativa. Rev Inic Cient Ext. 2019; 2(2): 96-101.

9. OMS. ORGANIZAÇÃO MUNDIAL DA SAÚDE. Draft: Global Strategy on Digital Health 2020-2024. (2020). Disponível em: https://www.who.int/docs/default-source/documents/gs4dh0c510c483a9a42b1834a8f4d276c6352.pdf. Acessado em: 07 de março de 2021.

10. OMS. ORGANIZAÇÃO MUNDIAL DA SAÚDE. Telemedicine, opportunities and developments in Member States: report on the second global survey on e-Health. 2009.2 Disponível em: https://apps.who.int/iris/bitstream/handle/10665/44497/9789241564144_eng.pdf?sequence=1\&isAllowed=y. Acessado em: 11 de agosto de 2020.

11. OPAS. ORGANIZACIÓN PANAMERICANA DE LA SALUD. Bases metodológicas para evaluar la viabilidad y el impacto de proyectos de telemedicina. Washington: OPAS; 2001, 138p. Disponível em: https://iris.paho.org/bitstream/handle/10665.2/784/9275323631.pdf?sequence=1\&isAllowed=y. Acessado em: 15 de agosto de 2020.

12. OPAS. ORGANIZACIÓN PANAMERICANA DE LA SALUD. Marco de Implementación de un Servicio de Telemedicina. Washington, DC: OPS, 2016, 82p. Disponível em: https://iris.paho.org/bitstream/handle/10665.2/28413/9789275319031_spa.pdf?sequence=6\&isAllowed=y. Acessado em: 08 de dezembro de 2020.

13. REZENDE EJC, et al. Souza C Ética e telessaúde: reflexões para uma prática segura. Rev Panam Salud Publica. $2010 ; 28(1): 58-65$.

14. SECOM - Secretaria de Comunicação. Secretaria de Estado de Planejamento, Desenvolvimento, Ciência, Tecnologia e Inovação - SEPLANCTI. Amazonas em Mapas. 1a Edição, 2016, 221p. Disponível em: http://www.sedecti.am.gov.br/wpcontent/uploads/2019/07/1a_Amazonas_em_Mapas_2015_em_novembro_de_2016.pdf. Acessado em: 10 de janeiro de 2021.

15. SILVA TPS, et al. Tele-educação em saúde da comunicação humana para o enfrentamento da tríplice endemia em Pernambuco, Brasil: um relato de experiência. Rev. CEFAC. 2020; 22 (3): e9519. 